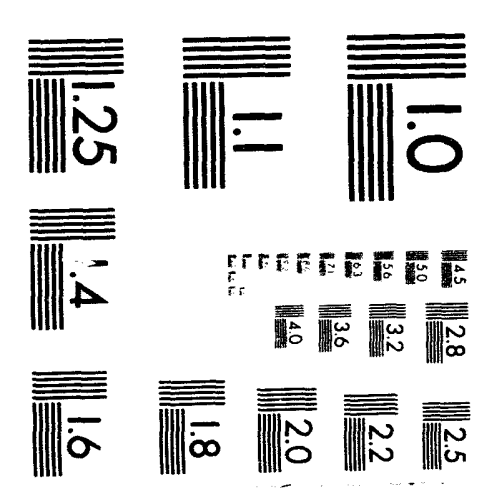



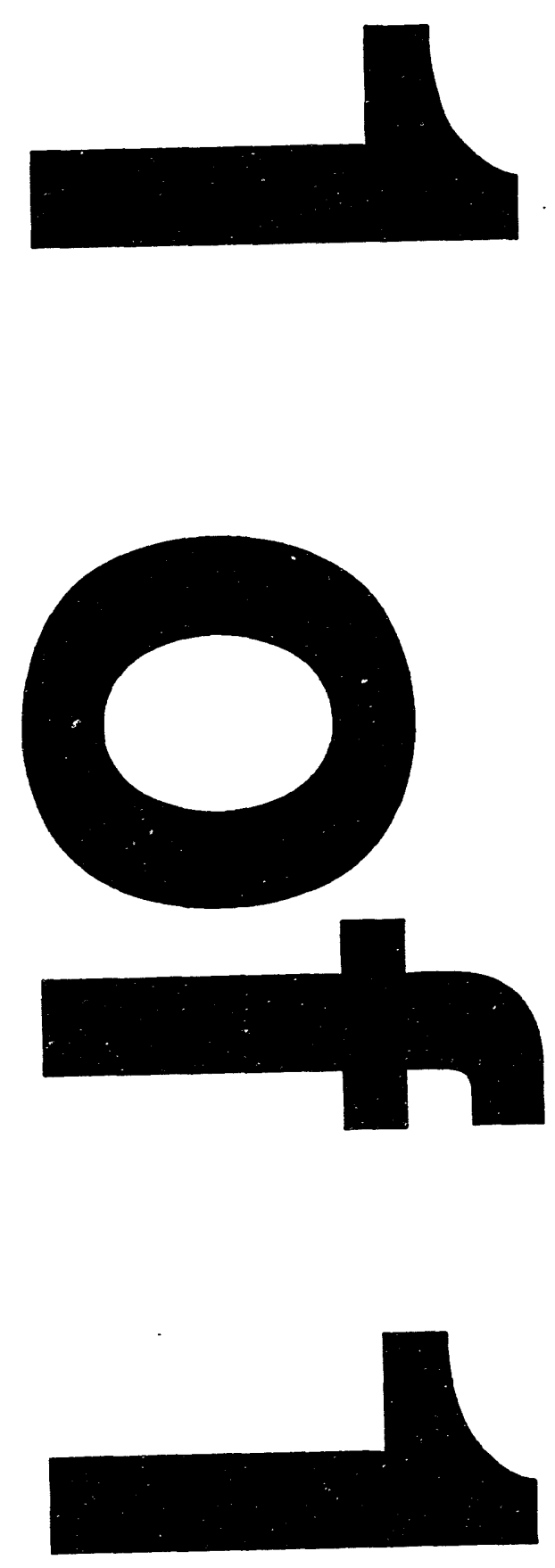


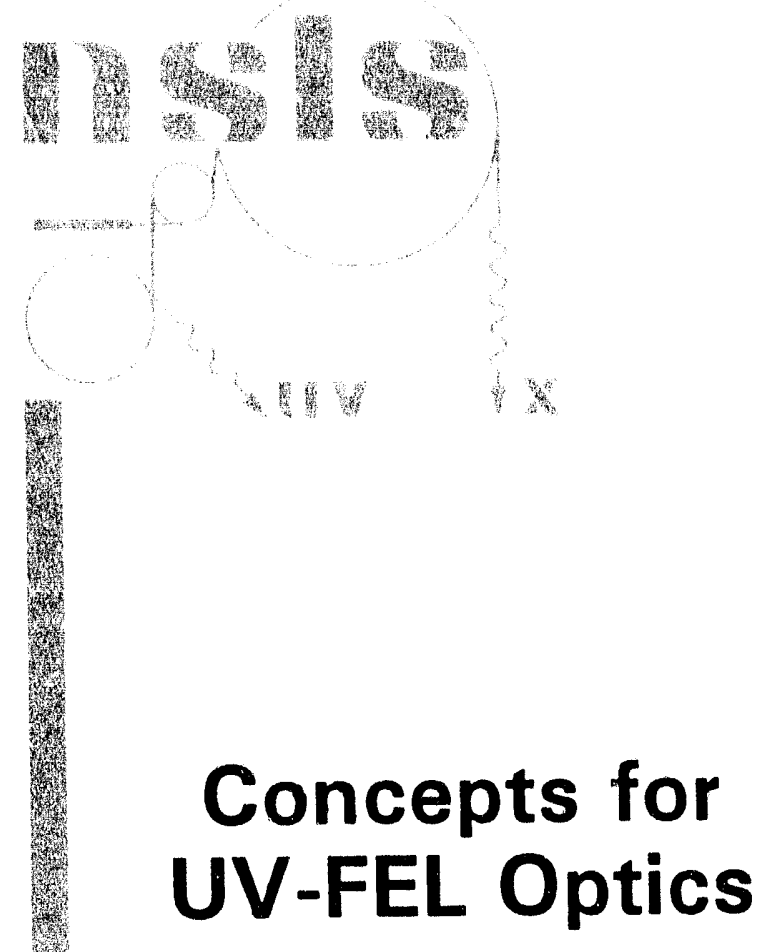

Brookhaven National Lab

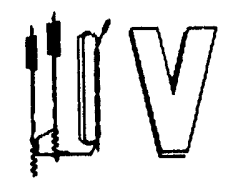

Free Electron Laser

Erik D. Johnson

November, 1993

Research Supported by the OFFICE OF BASIC ENERGY SCIENCES

\section{NATIONAL SYNCHROTRON LIGHT SOURCE}

BROOKHAVEN NATIONAL LABORATORY ASSOCIATED UNIVERSITIES, INC. 
This report was prepared as an account of work sponsored by an agency of the United States Government. Neither the United States Government nor any agency thereof, nor any of their employees, nor any of their contractors, subcontractors, or their employees, makes any warranty, express or implied, or assumes any legal liability or responsibility for the accuracy, completeness, or usefulness of any information, apparatus, product, or process disclosed, or represents that its use would not infringe privately owned rights. Reference herein to any specific commercial product, process, or service by trade name, trademark, manufacturer, or otherwise, does not necessarily constitute or imply its endorsement, recommendation, or favoring by the United States Government or any agency, contractor or subcontractor thereof. The views and opinions of authors expressed herein do not necessarily state or reflect those of the United States Government or any agency, contractor or subcontractor thereof. 


\title{
Concepts for UV-FEL Optics
}

\author{
Erik D. Johnson \\ National Synchrotron Light Source \\ Brookhaven National Laboratory \\ Upton New York USA 11973
}

\section{Brookhaven National Lab

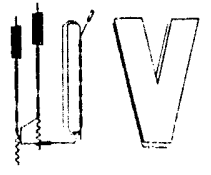 \\ Free Electron Laser}

\begin{abstract}
:
Brookhaven National Laboratory has developed a design for an ultra-violet free electron laser facility utilizing a seeded amplifier approach. Since the accelerator is a single pass device, resonator and outcoupler mirrors which are a difficult aspect of oscillator FEL designs are not required. The result is a source of high peak power VUV radiation with the mode structure, bandwidth and frequency stability of the input seed laser. The accelerator provides pulses of radiation at up to $10 \mathrm{kHz}$, so to maximize the utilization of the source, novel optical systems to share the radiation had to be developed. These include specialized alignment, beam transport, order sorting, and multiplexing optics. In addition, FEL on FEL pump-probe experiments are made possible by a variable optical delay of up to $10 \mathrm{~ns}$ operating in the wavelength range of 200 to $75 \mathrm{~nm}$. Some aspects of the FEL design are also described to clarify the constraints and choices for the optical system.
\end{abstract}

\section{INTRODUCTION:}

The proposal for an ultra-violet free electron laser (UV-FEL) grew from the realization that neither existing lasers or synchrotrons, nor the third generation synchrotron radiation sources now under construction address all of the needs of scientists interested in the ultraviolet region of the spectrum, particularly with respect to the combination of continuous wavelength selection, high peak power and short pulse duration [1]. The UV-FEL is a user facility designed to provide pico-second and sub-picosecond pulses of coherent ultraviolet radiation for wavelengths from 300 to $75 \mathrm{~nm}$ [2]. Pulse width will be variable from about $6 \mathrm{ps}$ to $\approx 200 \mathrm{fs}$, with repetition rates as high as $10^{4} \mathrm{~Hz}$, single pulse energies $>1 \mathrm{~mJ}$ and hence peak pulse power $>200 \mathrm{MW}$ and average beam power $>10 \mathrm{~W}$. The facility will be capable of "pumpprobe" experiments utilizing the FEL radiation with: 1) synchronized auxiliary lasers, 2) a second, independently tunable FEL beam, or 3) broad-spectrum, high-intensity $x$ rays from the adjacent National Synchrotron Light Source.

To understand the optical system design considerations, some description of the facility is required. Detailed information about the machine is beyond the scope of this paper, but overviews of the machine physics and design parameters are available $[3,4,5]$. Shown schematically in figure 1 , the UV-FEL consists of a high repetition rate recirculating superconducting linear accelerator which feeds pulses of electrons to two magnetic wigglers. Within these two devices, photons from tunable "conventional" lasers are frequency multiplied and amplified. By synchronously tuning the seed laser and modulating the energy of the electron beam, tuning of as much as 
$60 \%$ in wavelength is possible between alternating pulses supplied to different experimental stations, with Fourier transform limited resolution. The electrons for the FEL are generated by a high repetition rate $(10 \mathrm{kHz})$ laser photo-cathode electron gun which provides intense picosecond electron bunches (ca. 1-2 nC) that are further accelerated by the linac. However, many pump-probe experiments employing conventional auxiliary lasers cannot fully utilize all of the output of the FEL since the

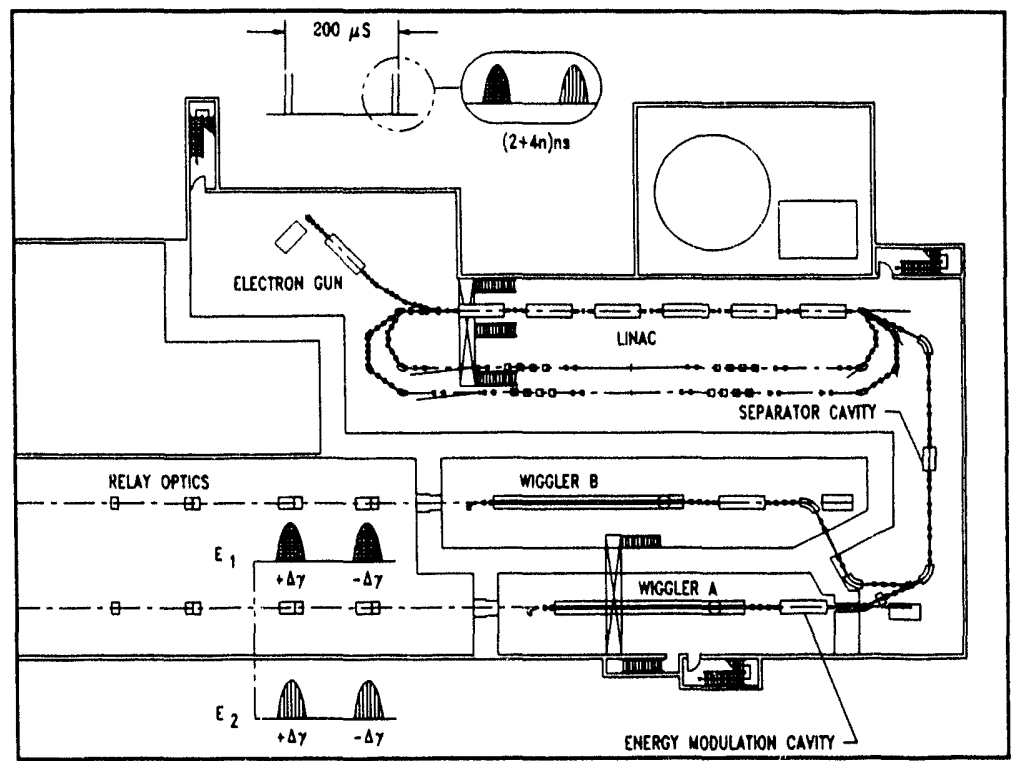

Figure 1 Schematic representation of UV-FEL accelerator and pulse distribution. repetition rates of these lasers are seldom greater than $1 \mathrm{kHz}$. Thus, the design provides for electron and photon beam multiplexing that allows for switching of alternating pulses between several users.

Since each user could require different wavelength radiation for their experiment, additional radio frequency ( $r f$ ) cavities were added before each wiggler to modulate the electron beam energy. By using several seed lasers coupled to the FEL through an optical switchyard, each user could control the tuning of their respective seed laser and, in concert with the energy modulation provided by the rf cavities, control the wavelength of the radiation delivered to the experiment. In addition to selecting the energy of the FEL radiation, the user could control the pulse duration of their seed laser, and hence the duration of the FEL radiation at their experiment. This arrangement allows the radiation from the FEL to be shared among up to four independent experimental stations simultaneously.

\section{OPTICS DESIGN:}

The optical system takes advantage of the unique characteristics of the FEL radiation, and the placement of the laboratory space roughly $5.35 \mathrm{~m}$ above the accelerator plant, as shown schematically in figure 2. This allows the UV radiation to be deflected up to each of the eight stations while keeping the experiments well out of the bremsstrahlung produced by the FEL. The beamlines are made with the largest possible included angles to minimize reflection losses. For the optics design, we make note of the fact that the apparent source size is essentially that of the electron beam $(0.2 \mathrm{~mm} \sigma)$, and that it is diffraction limited. Because the electron beam is continuously focused in the radiator and tapered sections of the FEL, we can regard the point of source divergence as being at the end of the wiggler. 
The operating parameters of the facility drive the choice of reflection optics and the materials which have been selected. Since the wavelength of the FEL radiation is below the LiF cutoff, conventional refraction optics such as lenses and prisms are unsatisfactory. Similarly, transmissiongratings and Fresnel zone plate structures have a strong wavelength dependance on their parameters, including focal length and efficiency,

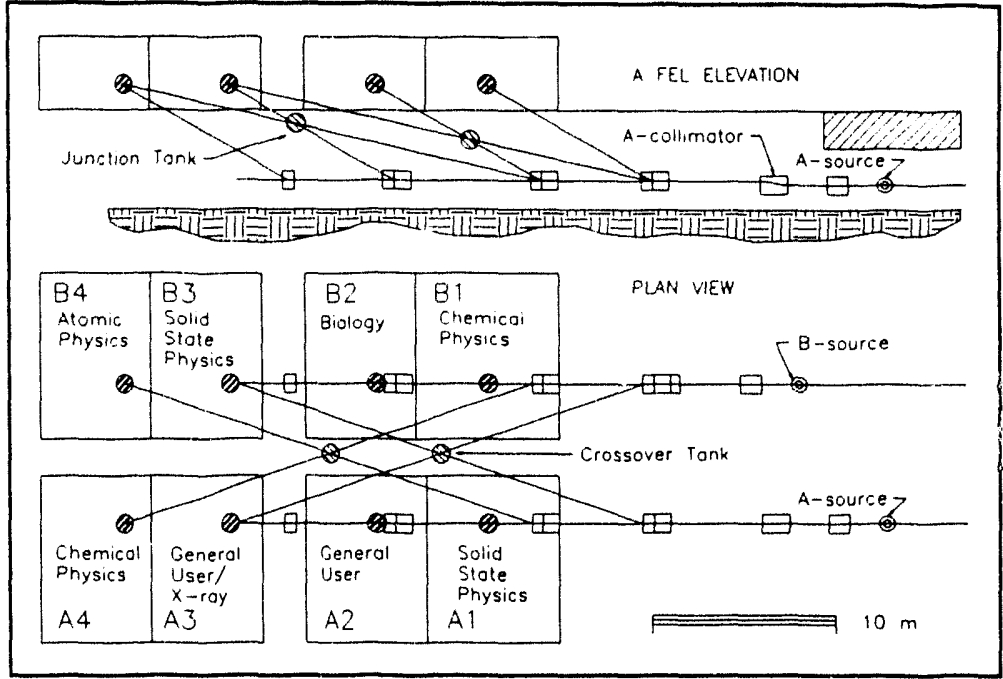

Figure 2 Schematic of optical paths from FEL to user laboratories. and are therefore also unsuited to the wide wavelength range operation of the facility. Incidence angles on reflection optics must also be carefully examined for this same reason. To maintain high reflectivity, contamination must be minimized since capping layers typically used above $125 \mathrm{~nm}$ such as $\mathrm{MgF}_{2}$ become highly absorbing at shorter wavelengths. In situ cleaning techniques $[6,7]$, and occasional recoating of optics will almost certainly be required [8]. These problems, familiar to designers of XUV optics [8,9], will be shared by essentially all of the optical elements utilized in the beamlines for the UV-FEL.

The collimation optics tank is the first element following the FEL and actually contains two mirrors, both fabricated from silicon, and overcoated with aluminum. The first is an off axis parabaloid with a focal length of 7 meters and an incidence angle of 6.25 degrees. The collimated beam is redirected to be parallel with the floor by a flat steering mirror. By taking the divergent beam to parallel rays, the actual displacement of subseruent optical systems with respect to the source has no effect on the beam collection efficiency. This allows identical elements to be used for each of the experimental stations.

As shown in figure 2, four laboratories are served directly by each wiggler line. Flat mirrors deflect the collimated radiation up to the lab, where it can be further conditioned as suits the experiment. If the initial flat mirror is placed directly in the FEL beam, only one user is served by each device. Although this type of operation may be required for some experiments, for many, the full repetition rate of the facility would be too high. A far more productive use of the electron beam can be made by pulse sharing, and taking advantage of the fast tuning capability provided by the accelerator to supply two (or more) experiments with pulses from the FEL.

This is accomplished by interposing a pair of parallel flat mirrors to produce a net displacement of the beam. The displaced beam is directed to the lab above by a deflection mirror. If the mirror pair is removed, the beam is not displaced and passes 
over the deflection mirror to a subsequent optical system. To move the mirrors in and out of the beam rapidly, several pairs of mirrors are arranged around the axis of a cylinder, and rotated in and out of position synchronously with, the r.f. clock of the accelerator. The spaces between reflecting pairs are open to allow the beam to pass unimpeded. The drive motor is based on a magnetic

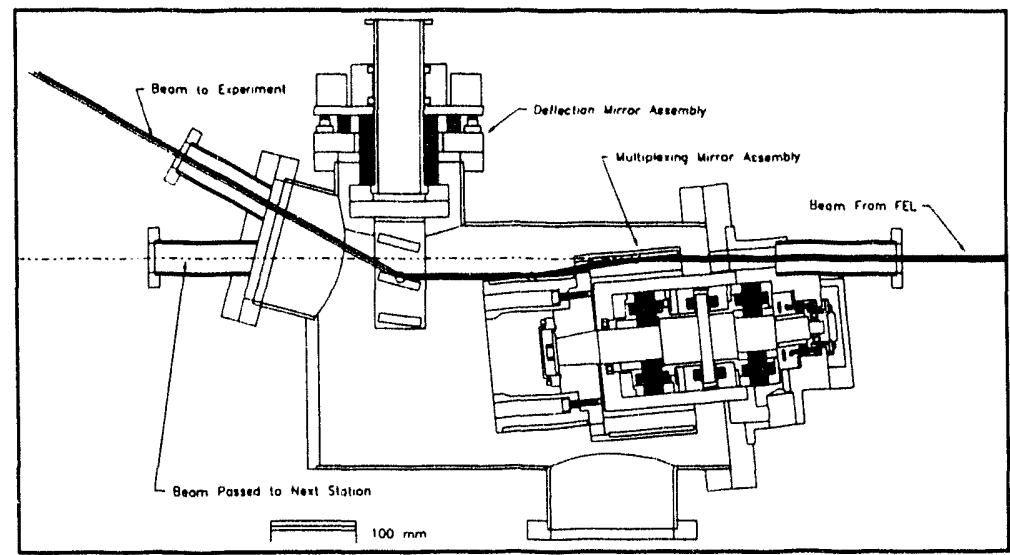

Figure 3 Outline drawing of beam multiplexing and steering system. bearing turbo-molecular pump with a nominal operating frequency of $400 \mathrm{~Hz}$. Using eight mirror pairs and eight open spaces, the switching rate is $6.4 \mathrm{kHz}$. For the $\mathrm{FEL}$ application $5 \mathrm{kHz}$ is required. It should be noted that commercially available motors of this type exist which operate above $1 \mathrm{kHz}$, so considerable increase in rate is possible. The choice of a magnetic or dry bearing system is motivated by the desire to minimize contamination of the mirror surfaces. The integrated system is shown in figure 3 with the multiplexing mirror assembly mounted on a 14 inch conflat flange, and also shows the deflection mirrors. As drawn, this includes positions for three different deflection mirrors in a ladder type configuration. For high energy beams, aluminum surface mirrors will be used. Silicon carbide or coated metal mirrors will be used where harmonic suppression is desired.

Although it is difficult to calculate, the third harmonic content in the beam is estimated to be the order of $10^{-3}$ of that in the fundamental. For certain experiments, such as excited state photoemission, this represents a useful intensity although it will be necessary to filter out the fundamental, since it could produce enough photocurrent to cause space charge problems. On the other hand experiments such as threshold spectroscopies require that the harmonic content be below $10^{-5}$ of the intensity in the fundamental. For some applications, the $10^{-3}$ harmonic content of the beam represents no difficulty, but high flux (and thus optical efficiency) are highly desirable.

Clearly to satisfy each of these requirements some type of monochromator or order sorting optics are required. This, coupled with the desire to make each of the stations flexible as well as identical, has lead us to develop a modified Czerny-Turner instrument which takes advantage of the precollimated beam.

The instrument takes advantage of the fact that the FEL is a quasimonochromatic source, so it need not have slits for the purpose of wavelength selection. Apertures, or in most cases the geometry of the optics suffice to select the desired diffracted order. This means that the last optical element, a parabolic mirror, can focus directly to the experimental station. Figure 4 schematically shows the arrangement of the components for the order sorting optics in the configuration for selecting high energy radiation. The input beam enters the instrument at an angle of 
29 degrees with respect to the horizontal plane where, for the case shown, it intercepts a plane grating. The first inside order diffracted beam falls on the parabolic focusing mirror which directs the output beam to the horizontal, and focuses at the experiment $1.5 \mathrm{~m}$ from the mirror pole. Several other components can also be seen, with functions which will be discussed shortly. These include four piane mirrors, four

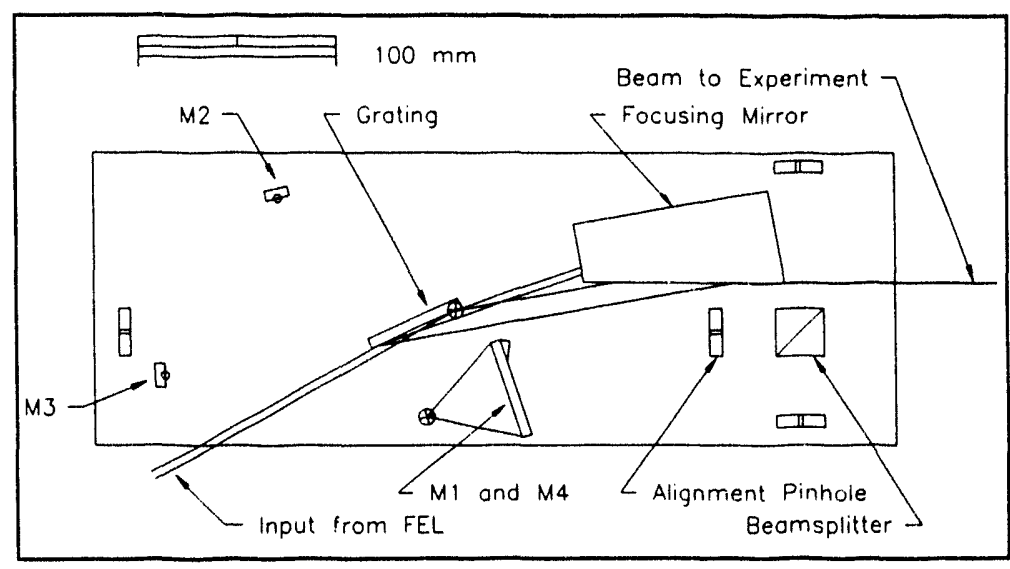

Figure 4 Schematic of order sorter and its basic components. alignment fiducial pinholes, and a stationary quartz beamsplitter, also for alignment purposes.

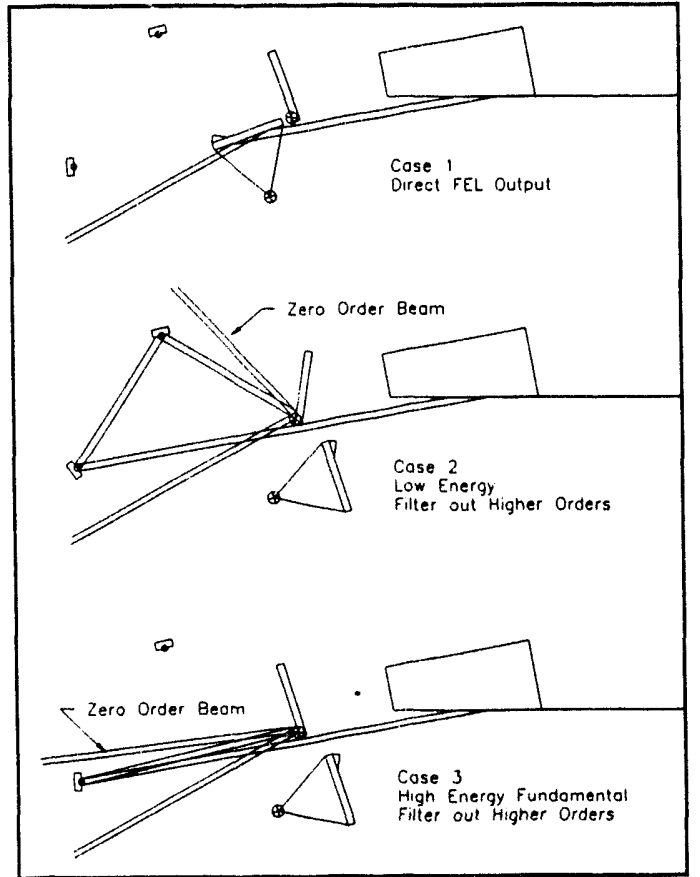

Figure 5 Order-sorter optical paths for functions described in text.
The case shown is actually only one of four configurations of the instrument to meet the needs of the experimental programs. The optical paths for the three additional cases are given in figure 5 . The first case is that of relaying the beam without filtering and with the highest possible efficiency to the experiment. To accomplish this, the grating is rotated out of the way, and the first mirror $M 1$, is rotated into place from the point indicated by the crossed circle. This mirror is a silicon flat 30 by $50 \mathrm{~mm}$ long with an included angle for the reflection of 161 degrees. The exit beam falls on the parabolic focusing mirror at a grazing incidence angle of 10 degrees.

The arrangement for the second case is designed to select radiation in the 125 to 200 $\mathrm{nm}$ range, while eliminating the FEL harmonics. In this configuration the beam is intercepted by the grating. It is made from silicon and ruled with 2400 lines $/ \mathrm{mm}$ with a laminar profile and a groove depth of approximately $100 \mathrm{~nm}$. This same grating is used for all the diffracting configurations, although it is rotated to significantly different incidence angles for each range. For this case, the included angle is 60 degrees, and the first inside diffracted order is incident on the M2 mirror which is a $\mathrm{MgF}_{2}$ coated Aluminum flat. The $\mathrm{M} 2$ mirror position is fixed during bench alignment, and has an included angle of reflection of 91.8 degrees. The reflected beam from the $M 2$ mirror strikes the $M 3$ mirror which is a SiC flat. The M3 mirror can 
be rotated via a sine bar mechanism in normal operation, but in this configuration the included angle of the reflection is set to 42.7 degrees which directs the output beam to the focusing mirror at its required angle of incidence.

The principle of operation for this arrangement is of course to make multiple reflections from mirror surfaces which have poor short wavelength reflectivity, and thereby reduce the harmonic intensity to a greater degree than the fundamental. The $\mathrm{MgF}_{2}$ has a fairly sharp cut off for wavelengths shorter than $125 \mathrm{~nm}$, and does most of the work in this situation. It should also be noted that the zero order beam (specular reflection) from the grating clears the $\mathrm{M} 2$ mirror, so a suitable photodiode placed in this beam could be used as an intensity monitor for signal normalization. To access radiation from 125 to $75 \mathrm{~nm}$, a similar strategy is employed to achieve harmonic attenuation, shown in figure 5 as case 3 .

In this configuration, the radiation is dispersed by the grating through an included angle of only 15.7 degrees. This brings the first diffracted inside order to the M3 mirror which is rotated to reflect its output beam to the focusing mirror at the proper angle. What this translates to is a reflection off the M3 mirror with an included angle of only 3.3 degrees. This close to normal incidence, the $\mathrm{SiC}$ mirror has a strong cutoff at about $60 \mathrm{~nm}$, so radiation shorter than this will be filtered out. Since the FEL should only produce second harmonic radiation by spontaneous emission, its intensitv will be much lower than the third harmonic which has a sufficiently short wavelength to be attenuated relative to the fundamental. At $125 \mathrm{~nm}$ this attenuation ratio is about 50, while at shorter wavelengths it is 1000 or more. Assuming an initial harmonic content of $10^{-3}$, this means that at the experiment, harmonic content the order of $10^{-5}$ or $10^{-6}$ could be achieved. This does however have a price since the intensity at the fundamental wavelength will be reduced to roughly 5 to $10 \%$ of that in the unconditioned beam. Still for many experiments such as threshold photoionization measurements, this loss in intensity is more than offset by gains in signal to background, so this mode of operation is well worth including in the beamline design.

The other extreme is embodied in the case 4 schematic (the optical path in figure 4) which shows the arrangement for selecting the third harmonic, while rejecting the fundamental. This is accomplished by making the first inside order of the diffracted radiation matched to the third harmonic. The fundamental is then channeled into the zero order beam, which enters a beam dump milled into the focusing mirror blank. Unfortunately this zero order beam most likely can not be used for signal normalization (as in the other configurations) since the intensity of the third harmonic generated in the FEL will be quite sensitive to charge in the electron beam, and coupling to the modes of the fundamental.

The structure of the actual instrument is shown in figure 6, which includes the monochromator, the vacuum chamber, and the alignment optics bench. The monochromator is built and pre-aligned on a plate which slides into the vacuum chamber and rests on stationary kinematic mounts. The support for these mounts 
couples through the vacuum chamber via bellows to a larger frame which also forms the support structure for the alignment laser and optics. This main frame is kinematically mounted on a stand and in effect isolated from the vacuum system and its pumping apparatus. During assembly the morochromator and alignment optics are set up on a bench without the vacuum chamiver. This facilitates easy adjustment of the stationary components and simplifies the design of their

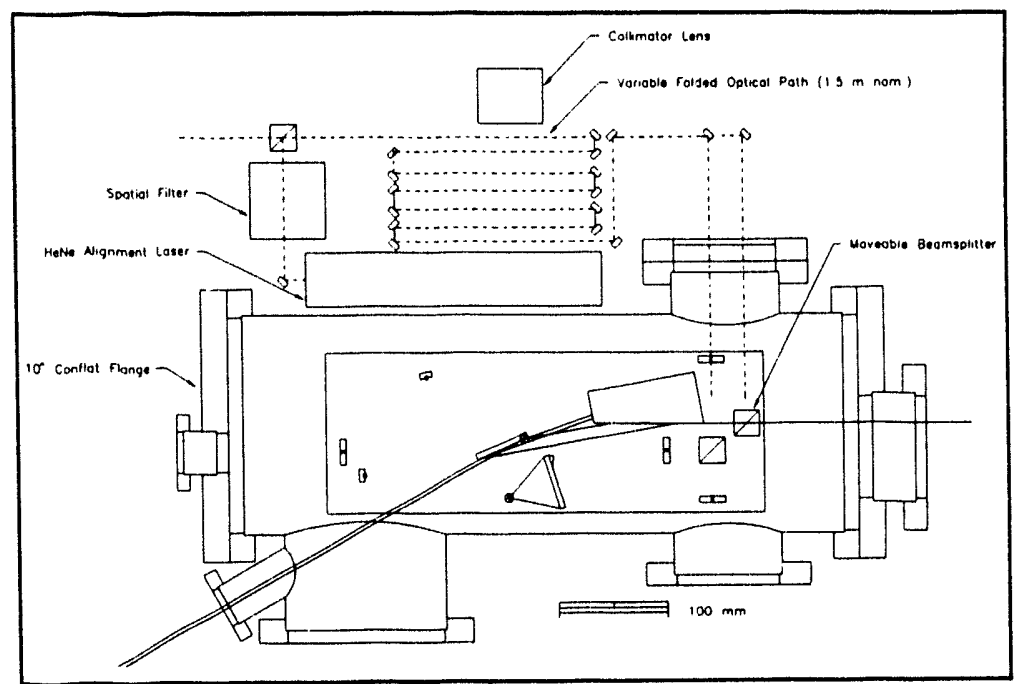

Figure 6 Layout of order-sorter with vacuum system and alignment tools. mounts since in-vacuum operation of these elements is not required.

For experiments which involve FEL on FEL beam configurations it may be necessary to introduce an optical delay to obtain the desired phase relationship between the pump and probe beams. A portion of the required delay can be made by changing the timing between the photocathode gun laser pulses. This is however an interval relationship because it must be in phase with the accelerator r.f. system. Delays of the form $t=2+4 n$ [ns] where $n$ is an integer are possible. This means the optical delay system must at a minimum provide a delay of $5 \mathrm{~ns}$ although $10 \mathrm{~ns}$ would be better since the operation of the entire facility might be briefly interrupted by changes in the gun laser timing. Since light takes 3.34 ns to traverse a meter distance in vacuum, the delay system must provide for path length changes of roughly 3 meters. The system must also provide the highest possible transmission. This can be achieved by providing a choice of either multifacet total external reflection geometry $[8,11,12]$ for short wavelength operation, or a 45 degree reflection geometry to minimize the number of reflections for longer wavelengths.

This dual approach is required to cover the FEL wavelength range since total external reflection optical systems have been demonstrated in the 35 to $100 \mathrm{~nm}$ regime [13] where most materials have unacceptably low normal incidence reflectivities. At wavelengths longer than about $100 \mathrm{~nm}$, the indices of refraction for candidate mirror materials become too close to unity, thus spoiling the high reflectivity provided by total external reflection. In this situation, the maximum overall transmission is achieved with the minimum number of reflections. In actual practice, both systems can easily be accommodated in the same frame for a folded trombone configuration as shown schematically in figure 7 .

The figure schematically shows two optical paths, one for each set of mirrors. In both cases, the output beam is displaced from the input beam and includes an 
angle difference of $1.3 \mathrm{mrad}$. This is introduced by steering the input beam on to the first element of the delay line with the relay mirror on the FEL floor level. The delay system is located in the laboratory where it is being used, so the relay optic is some 20 meters distant. In use, the delay system is lowered into the beam, so its exit beam is on the same optical line as would be followed by the undeviated beam from the FEL. The FEL beam is then deviated in angle

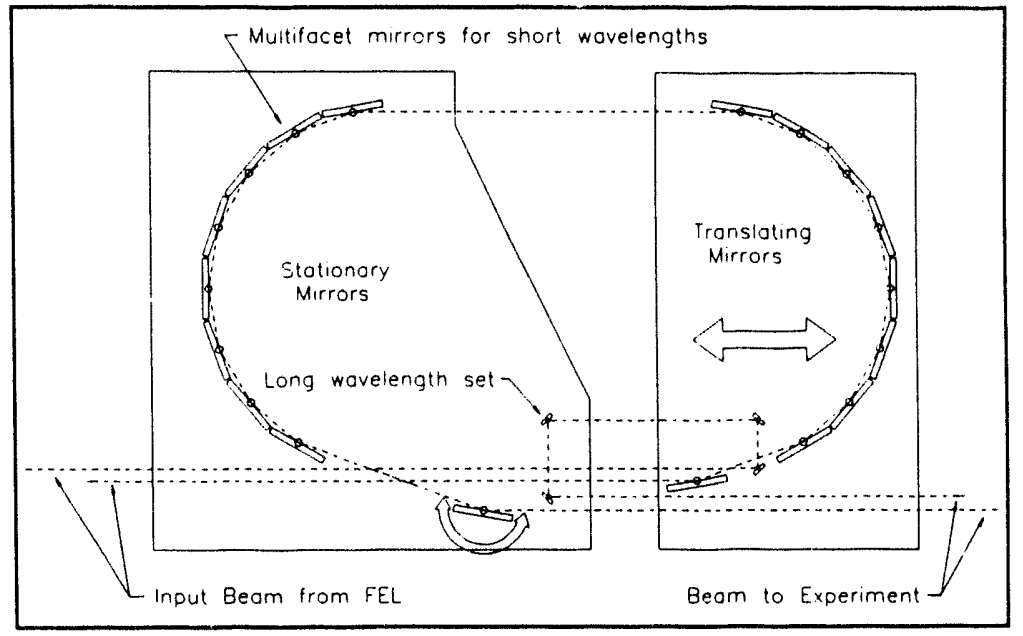

Figure 7 Schematic of optical components for optical delay line.

to fall on the surface of the first mirror on the translating mirror set. To change mirror sets for different wavelengths, the mirror assembly is translated vertically. Pointing errors are corrected by adjustments in roll and pitch of the last mirrors in the stationary sets. To assure proper steering of the beam, a beam position monitor of the type already in use at the NSLS will be employed [14]. Signals from this monitor will be used to steer the beam, and to correct the position of the translated mirror set to maintain the delay specified by the user.

The delay optical system is shown in figure 8 which includes the mounting and translation systems. The translating mirrors are mounted on a plate with three rollers on the back, two on the bottom, one on top. These follow a track made from centerless ground rods which are mounted on a support bench. The brackets holding the top rod provide some flexure to maintain a constant pressure on the assembly, while the tracking guidance is provided by the bottom rod. The mirror is translated with a relatively simple cable arrangement which pulls the mirror car along the track. After much consideration this scheme was adopted over more complex arrangements providing position encoding and precise movement since their precision would be lost in the noise generated by effects such as building thermal drift which introduce changes in path length and hence delay. For reliable operation feedback on the actual measured pulse separation will be required, for which our simple scheme is more than adequate. To provide 1 ps changes in delay, car motions 
of 150 microns are required, while the full $10 \mathrm{~ns}$ range requires 1.5 meter motion.

To integrate and align the individual optical systems, an alignment source is required. This instrument provides a visible beam which is coincident with the path of the UV-FEL beam for alignment purposes. It will be particularly valuable in ve:ifying the placement of the collimation system, and the multiplexing and relay optics. A $\mathrm{HeNe}$ laser source is used with a spatial filter and folded optical path chosen to simulate the placement of the point source provided by the FEL itself. The alignment system will be placed inside the FEL vault just after the beam dump magnet, so remote operation and observation are required.

An outline drawing of the system is given by figure 9 which shows the alignment system, a beamsplitter, a special prism, and a flat mirror to direct the laser source to the down stream optics. The top surface of the prism is silvered to provide an approximately $25 \%$ retroreflection of the incident laser beam and is used to assure colinearity of the alignment and FEL beams. When translated into the FEL beam the quartz from which the prism is made fluoresces in the

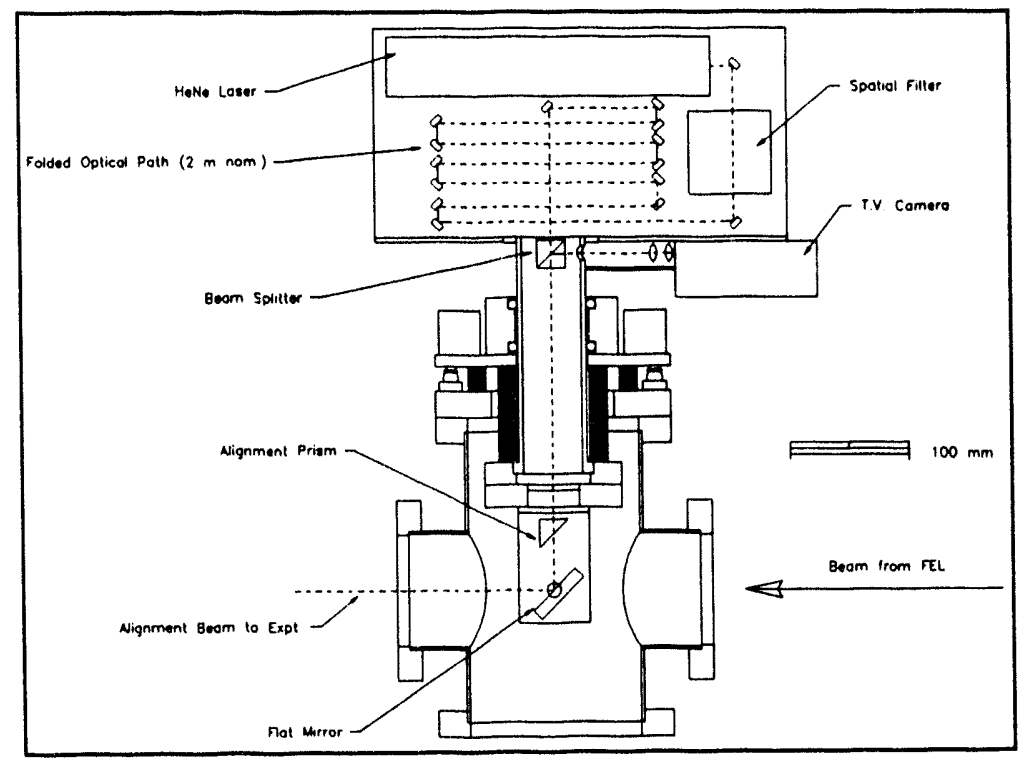

Figure 9 Layout of alignment system. visible. This blue radiation can be observed along with the retroreflected red from the alignment laser with the television camera and telescope shown in the drawing.

To send the alignment beam to the down stream optics, the whole assembly is translated up by the distance which separates the diagonal face of the prism and the flat mirror. Since these surfaces are parallel, the technique is insensitive to the exact position on the prism where the FEL and alignment beams are made to coincide. This may become important if after significant use the prism becomes opaque in the spot which has been exposed to the FEL due to the formation of color centers. By a simple translation of the alignment beam on the prism, new material is available for use. It may also be possible to anneal the damage out of the prism in situ, but the impact of this procedure on the alignment stability of the system will have to be evaluated.

When the system is not in use, the FEL beam passes between the mirror and prism. It should also be noted that if visible light were provided (say from ion gauges 
etc.) the alignment system is essentially a periscope which looks at the down stream components. Hopefully this feature will never be required, but experience indicates it may well be worth remembering during commissioning. The vacuum tank is fairly standard, and is pumped by a $220 \mathrm{l} / \mathrm{s}$ combination ion and sublimation pump. The whole assembly is provided with kinematic mounts for alignment and fiducial monuments on the manipulator table and shaft for survey.

\section{CONCLUDING REMARKS:}

The UV-FEL presents some unusual challenges for the design of its optical system. This has necessitated an approach which considers the entire system from the novel source and its properties to the diverse requirements of its users. The present work has attempted to outline in broad terms some of the conceptual designs developed to address these problems. The interested reader will find more detailed discussions in reference 5 . It is hoped that some of the concepts developed for this facility may be useful in other contexts such as the design of optical systems for photonic processing of materials, and in approaching the consideration of optical systems for other new radiation sources and applications.

\section{ACKNOWLEDGEMENTS:}

This work performed under the auspices of the U.S. Department of Energy, under contract DE-ACO2-76CH0OO16.

\section{REFERENCES:}

1. Report from the NSLS Workshop on Sources and Applications of High Intensity UV-VUV Light, E.D. Johnson and J.B. Hastings eds. January 1990, BNL informal report 45499.

2. L.H. Yu, Physical Review A, 44, pp. 5178-5193, Feb. 1990.

3. I. Ben-Zvi, L.F. DiMauro, S. Krinsky, M.G. White and L.H. Yu, "Proposed UVFEL User Facility at BNL", Proceedings of the 12th International FEL Conference, Sep. 1990, Paris, BNL-45161.

4. I. Ben-Zvi, L.F. DiMauro, S. Krinsky, M.G. White, L.H. Yu, K. Batchelor, A. Friedman, A.S. Fisher, H. Halama, G. Ingold, E.D. Johnson, S. Kramer, J.T. Rogers, L. Solomon, J. Wachtel and X. Zhang, "Proposed UV-FEL User Facility at BNL", Proceedings of the 13th International FEL Conference, Aug. 1991, Santa Fe NM, BNL-46673.

5. "UV-FEL Preliminary Design Report" I. Ben-Zvi ed. February 1993, BNL48565 . 
6 E.D. Johnson, S.L. Hulbert, R.F. Garrett, G.P. Williams, and M.L. Knotek, Rev. Sci. Instrum. 581042 (1987).

7 E.D. Johnson and R.F. Garrett, Nucl. Instr. and Meth. A266, 381 (1988).

8 B.E. Newnam et.al, "Development of an XUV-IR Free-Electron Laser User Facility for Scientific Research and Industrial Applications" Los Alamos publication number LA-UR-91-3532, submitted for the Proceedings of the Short Wavelength Radiation Sources Conference, SPIE Volume 1552, and references therein.

9 R. Reininger, Proceedings of 1989 CERN Accelerator School on Synchrotron Radiation and Free Electron Lasers, CERN 90-03, p401.

10 E.D. Johnson, S.L. Hulbert, and L.E. Berman, Am. Inst. Phys. Conf. Proceedings V249, 859 (1992), and references therein.

11 B.E. Newnam, "Multifacet, Metal Mirror Design for Extreme Ultraviolet and Soft $X$-ray Free-Electron Laser Resonators", Proceedings of Laser Induced Damage in Optical Materials: 1985, NBS Spec. Pub. 746, 261 (1988).

12 T.-Y. Hung and P.L. Hagelstein, "Investigations of Whisper Gallery Mirrors for Extreme Ultraviolet and Soft X-rays" IEEE Journal of Quantum Electronics 28 1376 (1992).

13 M.L. Scott, "Reflectance of Aluminum Reflectors in the Extreme Ultraviolet" OSA Proceedings on Short Wavelength Coherent Radiation: Generation and Applications, R.W.Falcone and J. Kirz eds. V2 p322 (1988).

14 E.D. Johnson and T. Oversluizen, Rev. Sci. Instrum 601947 (1989).

(Version 4.0 Last revision 18 October 1993) 

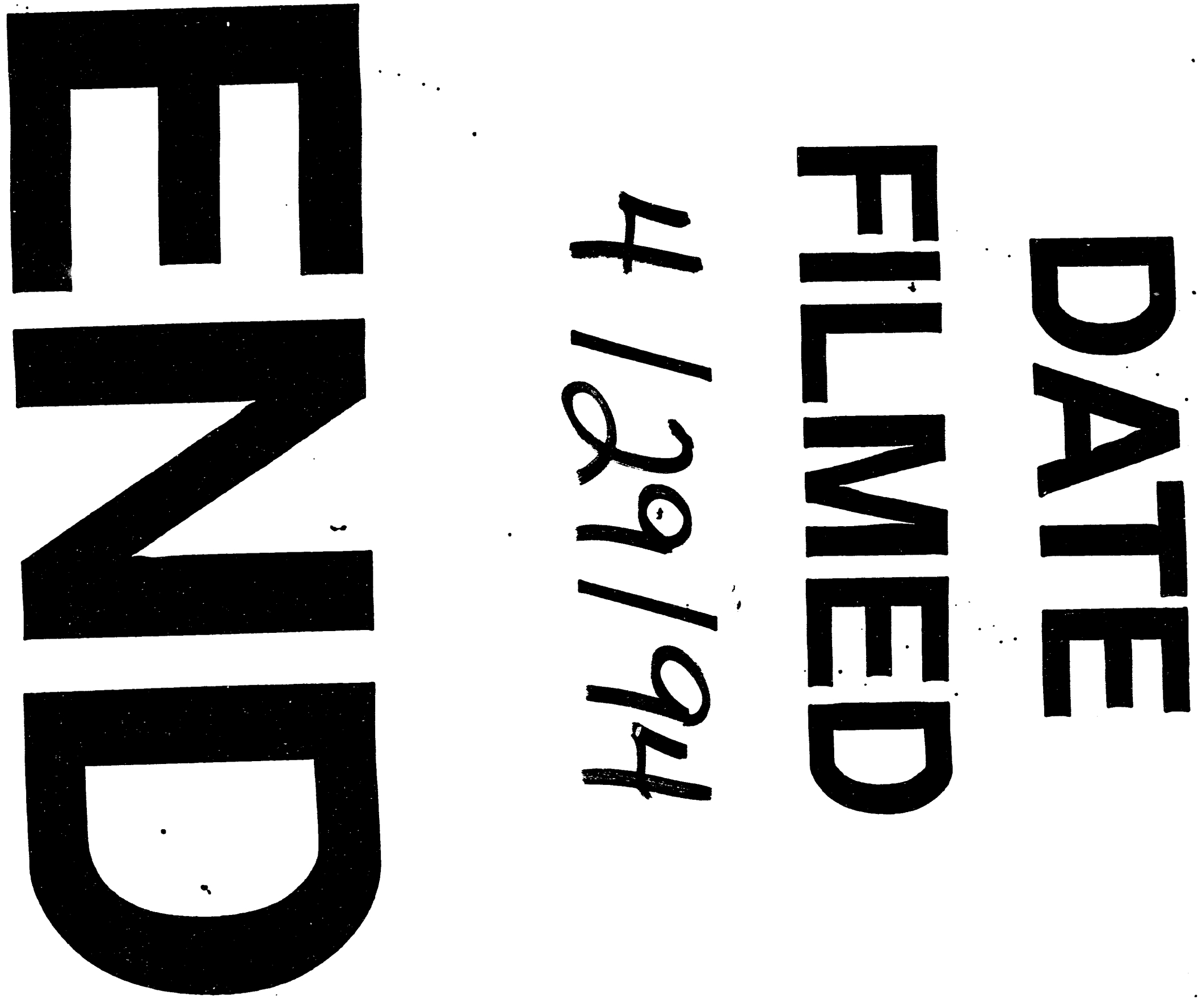
